\title{
Forceful dilatation under endoscopic control in the treatment of achalasia: a randomised trial of pneumatic versus metallic dilator
}

\author{
F Mearin, J R Armengol, L Chicharro, M Papo, A Balboa, J R Malagelada
}

\begin{abstract}
Forceful dilatation under endoscopic control is a well established treatment of achalasia; several different types of dilators can be used. This study prospectively compared the clinical and manometric efficacy of a single dilatation using two different dilators. Forty one patients were randomly assigned to forceful dilatation under endoscopic control with either a pneumatic dilator $(n=17)$ or a metallic dilator $(n=24)$. Thereafter, the patients received periodic clinical and manometric evaluation for one year (before and one, six, and 12 months after dilatation). One month after dilatation all but one of the subjects in each group had experienced good to excellent results and their clinical improvement persisted for the one year follow up. Two patients (one in each group) were perforated during the procedure and required surgical treatment. Recovery was uneventful in both cases. Resting lower oesophageal sphincter pressure (mean (SEM)) significantly and similarly decreased after both methods of dilatation (pneumatic dilator: before dilatation 37 (3) $\mathrm{mm} \mathbf{~ H g}$, one year after dilatation 18 (3) $\mathrm{mm} \mathrm{Hg}$; metallic dilator: before dilatation 34 (2) $\mathrm{mm} \mathrm{Hg}$, one year after dilatation 17 (3) $\mathbf{~ m m ~ H g ; ~}$ $p<0.05$ for both). It is concluded that in the treatment of achalasia a single dilatation under endoscopic control with either pneumatic or metallic dilator yield comparable clinical and manometric results and similar complication rates. The use of one or other dilator should depend more on the preference and experience of the endoscopist than on the type of device.
\end{abstract}

(Gut 1994; 35: 1360-1362)

Digestive System

Research Unit,

Hospital General

Universitari Vall

d'Hebron,

Autonomous

University of

Barcelona, Spain

F Mearin

J R Armengol

L Chicharro

M Papo

A Balboa

J R Malagelada

Correspondence to: Dr F Mearin, Digestive System Research Unit, Hospital General Vall d'Hebron, 08035-Barcelona, Spain

Accepted for publication 9 February 1994

Achalasia is a primary oesophageal motility disorder characterised by aperistalsis of the oesophageal body and impaired relaxation in response to swallowing of an often hypertonic lower oesophageal sphincter (LOS). ${ }^{1}$ As the propulsive force of the oesophagus cannot be restored, treatment aims at decreasing resistance to flow at the oesophagogastric junction. This may be achieved by decreasing LOS tone pharmacologically ${ }^{2}$ or more definitively by disrupting the circular muscular fibres of the sphincter, either by forceful dilatation or by surgery. ${ }^{34}$ Results depend on the method used, on the expertise of the medical team, and on the length of follow up. Published reports show that good clinical results are obtained from 72 to $92 \%$ of patients having surgery ${ }^{6}$ and from 50 to $77 \%$ of those having dilatation. ${ }^{3} 7$

In this study we have prospectively compared the therapeutic efficacy of two different dilators used by us in clinical practice. Forty one achalasia patients were randomly assigned to forceful dilatation under endoscopic control using either a pneumatic dilator or a metallic dilator. Patients were followed up clinically and manometrically for the subsequent year (before, and one, six, and 12 months after dilatation).

\section{Methods}

\section{PATIENTS}

Forty one consecutive patients fulfilling the clinical, radiological, endoscopic, and manometric diagnosis of achalasia $^{1}$ who were previously untreated entered the study. Patients were randomly assigned to two different methods of forceful dilatation: 17 patients to receive dilatation with a pneumatic device and 24 with a metallic device. Patients with sigmoid dilatation of the oesophagus were not excluded (two in the pneumatic dilatation group and three in the metallic dilatation group). The Table shows that the clinical features of each group were similar.

\section{CLINICAL EVALUATION}

A careful clinical evaluation was undertaken before treatment and one, six, and 12 months after dilatation using a standard questionnaire. Severity of dysphagia, regurgitation, chest pain, heartburn, and weight loss were assessed. Results of treatment were classified as excellent, good, moderate or poor according to the criteria published by Vantrappen and Hellemans. ${ }^{3}$
Clinical data and manometric findings before dilatation

\begin{tabular}{lll}
\hline & $\begin{array}{l}\text { Pneumatic } \\
\text { dilatation } \\
(n=17)\end{array}$ & $\begin{array}{l}\text { Metallic } \\
\text { dilatation } \\
(n=24)\end{array}$ \\
\hline Age (y) & $52(5)$ & $50(2)$ \\
Range & $23-90$ & $12-81$ \\
Sex (male/female) & $9 / 8$ & $16 / 8$ \\
Duration of symptoms (months) & $49(10)$ & $48(8)$ \\
Symptom severity (II, III, IV) & $1,3,13$ & $1,9,14$ \\
LOS pressure (mm Hg) & $37(3)$ & $34(2)$ \\
LOS relaxation (\%) & $33(5)$ & $33(4)$ \\
Oesophageal wave amplitude (mm Hg) & $24(4)$ & $28(6)$
\end{tabular}

Data shown as mean (SEM). * (II: mild; III: moderate; IV: severe). 
MANOMETRIC EVALUATION

Oesophageal intraluminal pressures were measured using a four lumen polyvinyl tube $(0.9 \mathrm{~mm}$ internal diameter) with its orifices spaced at $5 \mathrm{~cm}$ intervals along the distal part of the tube. The lateral opening manometric catheters were radially oriented. They were perfused with distilled water at $0.3 \mathrm{ml} / \mathrm{min}$ through a pneumohydraulic system. Respiration and swallowing were monitored by flexible bellows placed around the chest and neck respectively. Oesophageal pressure activity was recorded graphically on a paper polygraph (model 1600; MFE Corporation, Salem, NH). Studies were performed in the supine position after oral passage of the manometric tube. Resting LOS pressure was determined by the station pull through technique using intragastric pressure as the zero reference. The mean (SEM) of the eight values obtained during two pull throughs of the four lumen catheters was obtained. Manometric tracings were analysed by one of the investigators (FM) who was unaware of the dilatation method used.

\section{FORCEFUL DILATATION TECHNIQUE}

All dilatation procedures were performed by the same investigator (JRA) and only one dilatation was performed in each patient. In all cases dilatation was carried out after 24 hours of liquid diet and an overnight fast. Patients were given intravenous benzodiazepine before the procedure. Four hours after dilatation an $x$ ray of the chest was obtained to exclude perforation. Patients remained in hospital for observation for 24 hours and were then discharged.

Pneumatic dilatation was performed using a Witzel dilator. ${ }^{8}$ This dilator consists of a polyvinyl tube, $20 \mathrm{~cm}$ in length, surrounded by a $15 \mathrm{~cm}$ long polyurethane balloon connected to an external inflation device. Maximal balloon diameter is $4 \mathrm{~cm}$ at a $300 \mathrm{~mm} \mathrm{Hg}$

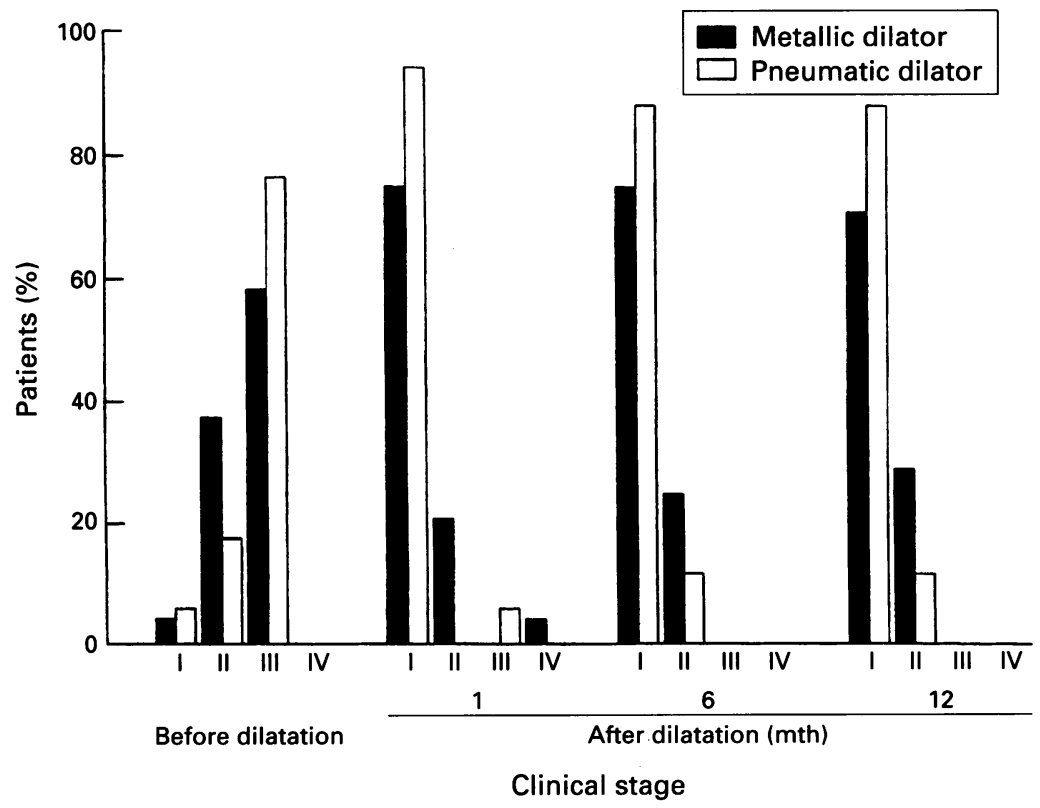

Figure 1: Clinical follow up one, six, and 12 months after dilatation. Results are classified as excellent (I), good (II), moderate (III), or poor (IV) according to the criteria of Vantrappen and Hellemans (ref 3). inflation pressure. The dilator was attached with rubber bands to a small calibre gastroscope (Olympus GIF, P). The dilator and gastroscope were introduced through the mouth and advanced until the endoscope tip was inside the stomach. The tip of the endoscope was then retroflexed so that the distal end of the balloon could be visualised at the gastrooesophageal junction. The endoscope was then further advanced until the midpoint of the balloon coincided with the gastro-oesophageal junction. At this point the balloon was gradually inflated to a pressure of $300 \mathrm{~mm} \mathrm{Hg}$ in one minute and maximal pressure was then maintained during three additional minutes.

Metallic dilatation was performed using a modified Stark dilator. ${ }^{9}$ This dilator is a mechanical device $10 \mathrm{~cm}$ long with four expanding metal arms. The maximal diameter of the dilating device once fully extended is 3.5 $\mathrm{cm}$. Dilatation was performed under endoscopic control in the following manner. The gastroscope was introduced and advanced until it reached the lower part of the oesophagus and the oesophagogastric junction was visualised. The dilator was then introduced, also through the mouth, and placed under direct vision across the gastro-oesophageal junction. The tip of the endoscope was advanced into the stomach and retroflexed to ensure the correct positioning of the dilator. At this point the metal arms of the dilator were gradually opened to the maximal diameter. This manoeuvre was repeated twice.

\section{Results}

Before treatment, the severity of symptoms and the oesophageal manometric findings were similar in both groups of patients (Table).

Shortly after treatment, symptoms were significantly improved in patients treated with either dilator; one month after endoscopic dilatation all but one of the subjects in each group had experienced good or excellent results. Such clinical improvement was sustained for the entire one year follow up period (Fig 1). No significant difference in the percentage of patients with excellent results (clinical stage I) was seen between the two groups at any time during follow up, although the pneumatic dilatation did slightly better. In the two unsuccessful cases, one of whom experienced only moderate and the other poor results, a second endoscopic dilatation (this time using the metallic dilator in both cases) was performed obtaining good clinical results.

Two patients had an oesophageal perforation during the dilatation procedure: one with the pneumatic dilator and the other with the metallic dilator. Both were treated surgically with simple closure of the perforation without myotomy. One patient experienced an upper gut haemorrhage after metallic dilatation and required transfusion of two blood units, and was treated medically. Recoveries were uneventful, and all patients were followed up clinically and manometrically, verifying excellent clinical results at one, six, and 12 months. During the one year follow up one 


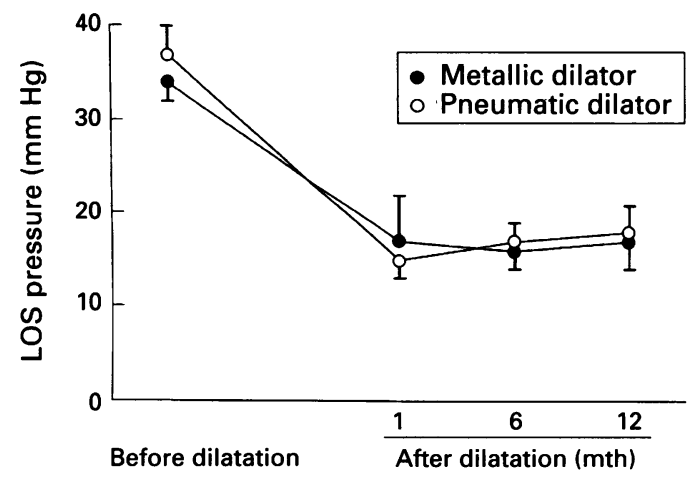

Figure 2: Lower oesophageal sphincter (LOS) pressure in achalasia patients before dilatation and during the one year after dilatation follow up.

patient of the pneumatic dilator group and two patients of the metallic dilator group complained of mild transient heartburn, which was well controlled with antacids.

Resting LOS pressure significantly and similarly decreased after both methods of dilatation (Fig 2). These manometric results were also maintained during the one year follow up. No differences in the manometric results were seen between pneumatic and metallic dilatation.

\section{Discussion}

Forceful dilatation is a well established treatment of achalasia. Whether it is preferable to surgical myotomy or not is a matter of debate. ${ }^{10}$ Both therapeutic procedures, dilatation and surgery, diminish oesophagogastric resistance to flow by disrupting the circular muscular fibres of the gastro-oesophageal junction. This is performed under direct vision during surgical oesophagomyotomy whereas the rupture of the muscle is blind during forceful dilatation. Surgery achieves a more complete degree of myotomy than dilatation as shown by comparing LOS pressures after both treatments. ${ }^{4}$ The smaller reduction in pressure obtained with dilatation, however, seems to be sufficient in most patients to improve their dysphagia. ${ }^{11} 12$ Moreover, forceful dilatation can normalise the mechanical and elastic properties of the gastrooesophageal junction as shown in vivo using a pneumatic resistometer. ${ }^{13}$ Dilatation has the additional advantage of being a brief procedure which, in most cases permits discharge of the patient within 24 hours. It has the disadvantage of carrying an associated risk of oesophageal perforation, which has been estimated to range from 1 to $6 \% .^{3}{ }^{12} 14$ The perforation rate in this study was $4.9 \%$, a figure slightly higher than our overall institutional experience rate of $3 \cdot 1 \%$. Whether the rather high perforation rate of this study results from the combined use of endoscopy and dilatation or to the small number of patients is difficult to ascertain. Perforation is a serious complication, which should be explained to the patient before the procedure but recognised early and managed effectively either with conservative or surgical treatment it carries a low death risk. ${ }^{15}$ In fact, non-complicated oesophageal perforation from dilatation in achalasia can be safely and effectively treated medically: nothing by mouth, total parenteral nutrition, and broad spectrum antibiotics. ${ }^{16}$ Both of our patients who suffered this complication survived without apparent sequelae.

In this study we have shown that a single forceful dilatation, either pneumatic or metallic, provides sustained clinical success at one year follow up in about $95 \%$ of cases. Similar results were obtained with either type of dilatation although a slightly higher percentage of excellent results were achieved with the pneumatic dilator. We cannot exclude a type 2 statistical error for the lack of significant statistical difference, given the comparatively small number of patients in the study. In our study we compared two different size dilators, a $40 \mathrm{~mm}$ diameter balloon dilator with a $35 \mathrm{~mm}$ metal dilator. This was because our goal was to compare techniques that we had previously standardised in our unit. In this paper we also describe how to perform a forceful dilatation with the Stark device under endoscopic control. This procedure, similar to that of pneumatic dilatation, in terms of its effectiveness permits an accurate placement of the dilator across the gastro-oesophageal junction and has the additional advantage of facilitating immediate diagnosis of perforation when it occurs.

In summary, this randomised trial comparing pneumatic versus metallic dilator in a single forceful dilatation under endoscopic control for the treatment of achalasia shows that for a one year follow up both methods have similar clinical and manometric results and similar complication rates. The use of one or other dilator should depend more on the preference and experience of the endoscopist rather than on the type of device.

1 Vantrappen G, Hellemans J. Achalasia. In: Vantrappen G, Hellemans J, eds. Diseases of the esophagus. Berlin Springer-Verlag, 1975: 287-354.

2 Bortolotti M, Labo G. Clinical and manometric effects of nifedipine in patients with esophageal achalasia. Gastroenterology 1981; 80: 39-44.

3 Vantrappen G, Hellemans J. Treatment of achalasia and related motor disorders. Gastroenterology 1980; 79: related $144-54$.

4 Csendes A, Braghetto I, Henriquez A, Cortés C. Late results of a prospective randomized study comparing forceful dilatation and oesophagomyotomy in patients with achalasia. Gut 1989; 30: 299-304.

5 Donahue PE, Samuelson S, Schlesinger PK, Bombek C, Nyhus LM. Achalasia of the esophagus. Treatment controversies and the method of choice. Ann Surg 1986; 203: 505-11.

6 Csendes A, Braghetto I, Mascaró J, Henriquez A. Late subjective and objective evaluation of the results of esophagomyotomy in 100 patients with achalasia of the esophagus. Surgery 1988; 104: 469-75.

7 Sauer L, Pellegrini C, Way LW. The treatment of achalasia. A current perspective. Arch Surg 1989; 124: 929-32.

$8 \mathrm{Witzel}$ L. Treatment of achalasia with a pneumatic dilator attached to a gastroscope. Endoscopy 1981; 79: 144-54. attached to a gastroscope. Endoscopy 1981; 79: 144-54.
Utkin V. Resultatsvergleich der kardiospasmus therapie. Zentralbl Chir 1973; 98: 743-7.

10 Vantrappen G, Janssens J. To dilate or to operate? That is the question. Gut 1983; 24: 1013-9.

11 Vantrappen G, Hellemans J, Deloof W, Valembois $P$, Vandenbrooke J. Treatment of achalasia with pneumatic dilatations. Gut 1971; 12: 268-75.

12 Fellows IW, Ogilvie AL, Atkinson M. Pneumatic dilatation in achalasia. Gut 1983; 24: 1020-3.

13 Mearin F, Zacchi P, Armengol J-R, Vallescar R, Malagelada $\mathrm{J}-\mathrm{R}$. Restored dynamic properties of the gastroesophagea junction in achalasia after forceful endoscopic dilatation. Gastroenterology 1991; 100: A123.

14 Benedict EB. Bougienage, forceful dilatation and surgery in the treatment of achalasia. Surg Gynecol Oncol 1964; 2: the treatm.

15 Shaffer HA, Valenzuela G, Mittal RK. Esophageal perforation. A reassessment of the criteria for choosing medical or tion. A reassessment of the criteria for choosing medical
surgical therapy. Arch Intern Med 1992; 152: 757-61.

16 Swedlund A, Traube M, Siskind BN, McCallum RW Nonsurgical management of esophageal perforation from pneumatic dilatation in achalasia. Dig Dis Sci 1989; 34: 379-84. 